\title{
Reemission spectra and inelastic processes at interaction of attosecond and shorter duration electromagnetic pulses with atoms
}

\author{
D.N. Makarov*, and V.I. Matveev \\ M.V. Lomonosov Northern (Arctic) Federal University, 163002 Arkhangelsk, Russia
}

\begin{abstract}
Inelastic processes and the reemission of attosecond and shorter electromagnetic pulses by atoms have been considered within the analytical solution of the Schrödinger equation in the sudden perturbation approximation. A method of calculations with the exact inclusion of spatial inhomogeneity of the field of an ultrashort pulse and the momenta of photons in the reemission processes has been developed. The probabilities of inelastic processes and spectra of reemission of ultrashort electromagnetic pulses by one- and many-electron atoms have been calculated. The results have been presented in the form of analytical formulas.
\end{abstract}

Progress currently achieved in the methods of generation of short and ultrashort electromagnetic pulses has allowed overcoming the "femtosecond limit" and obtaining pulses with a duration of several tens of attoseconds. As a result, attosecond physics appeared (see, e.g., reviews [1-5]) and it became possible to observe atomic phenomena on a real time scale. The processes accompanying the interaction of ultrashort pulses with atoms are usually described within the following approaches. For pulses shorter than the characteristic atomic time, but longer than attosecond pulses, it is appropriate to use approaches based on the Magnus expansion for the evolution operator. Only the first several terms in the expansion of the evolution operator [6] are currently used in calculations. In these calculations, the field of the pulse is assumed to be spatially uniform at the dimensions of a target $[7,8]$. The sudden perturbation approximation is appropriate for attosecond and shorter pulses. The possibilities of generation of pulses much shorter than attosecond ones are actively discussed (see, e.g., $[9,10])$. The exact solution of the Schrödinger equation in the sudden perturbation approximation for the interaction of attosecond and shorter electromagnetic pulses with multielectron atoms has been obtained in [11]. It is usually accepted [12] that the integral of the electric field strength of a laser source in vacuum with respect to time is zero. The description of the interaction of an atomic electron in the sudden perturbation approximation implies that the electron is free during the action of the field of the pulse; consequently, the absorption of laser radiation in vacuum is significantly nonlinear with respect to the field. The exact solution obtained in [11] makes it possible to calculate such effects.

\footnotetext{
*Corresponding authord.makarov@narfu.ru
} 
In this work, a method for calculating inelastic processes and spectra of reemission of extremely short electromagnetic pulses by atoms is developed on the basis of the exact solution of the Schrödinger equation in the sudden perturbation approximation obtained in [11]. In the cases under consideration, it is assumed that the duration of such pulses is much shorter than the characteristic atomic time. Thus, we consider attosecond and shorter electromagnetic pulses. The developed method allows the exact inclusion of the spatial inhomogeneity of the field of the electromagnetic pulse and the momenta of photons in the processes of reemission. The dipole approximation is not used. The field of the incident pulse is taken into account exactly in the sudden perturbation approximation, whereas the emission of a photon is described in perturbation theory. It should be noted that we are speaking of the emission of one photon by all atomic electrons during the action of a sudden perturbation. The reemission spectra include incoherent (proportional to $\mathrm{N}$ ) and coherent (proportional to N2) parts, where $\mathrm{N}$ is the number of electrons in the atom. The results are presented as simple analytical formulas containing several coefficients and screening parameters tabulated [13] for all atoms (with nuclear charges $1 \leq Z \leq 92$ ) with electron densities described by the well-known Dirac-Hartree-Fock-Slater model. By way of example, the spectra of reemission of ultrashort electromagnetic field Gaussian pulses by carbon, silicon, germanium, tin, and lead atoms are calculated. The method can be used not only for a Gaussian pulse, but also for any other pulse, provided that the sudden perturbation approximation is applicable. The choice of the Gaussian pulse is convenient because it allows following the transition from the field whose the integral of the electric field strength of the ultrashort pulse with respect to time is nonzero to the field with zero integral at the passage to limit. As an illustration, we numerically calculated the total probability of all inelastic processes in the hydrogen-like atom by using Gaussian pulse. Obviously, our method also permits direct generalization to molecular targets.

This work was supported by the Ministry of Education and Science of the Russian Federation (state assignment no. 3.1726.2014/K) and by the Council of the President of the Russian Federation for Support of Young Scientists and Leading Scientific Schools (project no. SP-1800.2015.1).

\section{References}

1. F. Krausz, M. Ivanov, Rev. Mod. Phys. 81, 163 (2009)

2. P.P. Agostini, L.F. DiMauro, Rep. Prog. Phys. 67, 813 (2004)

3. P.V. Corkit, F. Krausz, Nat. Phys. 3, 381 (2007)

4. V. Astapenko, Interaction of Ultrashort Electromagnetic Pulses with Matter, Springer Briefs in Physics (Springer, Heidelberg, 2013)

5. A.M. Zheltikov, Phys. Usp. 54, 29 (2011)

6. A.M. Dykhne, G.L. Yudin, Sov. Phys. Usp. 21, 549 (1978)

7. M. Klaiber, D. Dimitrovski, J.S. Briggs, Phys. Rev. A 79, 043402 (2009)

8. D. Dimitrovski, M. Ferre, L.B. Madsen, Phys. Rev. A 80, 053412 (2009)

9. A.E. Kaplan, Lasers Eng. 24, 3 (2013)

10. G. Mourou, S. Mironov, E. Khazanov, A. Sergeev, Eur. Phys. J. 223, 1181 (2014)

11. D.N. Makarov, V.I. Matveev, JETP Lett. 103, 415 (2016)

12. B.M. Karnakov, V.D. Mur, S.V. Popruzhenko, V.S. Popov, Phys. Usp. 58, 3 (2015)

13. F. Salvat, J.D. Martinez, R. Mayol, J. Parellada, Phys. Rev. A 36, 467 (1987) 